\title{
Poster Abstract
}

\section{Cytokinin homeostasis and its application in seed yield in brassica}

\author{
D. O'KEEFE, J. SONG and P. JAMESON \\ School of Biological Sciences, University of Canterbury, Private Bag 4800, Christchurch, \\ New Zealand \\ paula.jameson@canterbury.ac.nz
}

\begin{abstract}
Cytokinins are hormones that regulate many developmental and physiological processes in plants. Seed development is partly controlled by the balance of cytokinin biosynthetic and metabolism genes. In the normal course of seed development the levels of active cytokinins decrease significantly. To determine if the levels of cytokinin are controlled by degradation by cytokinin oxidase in
\end{abstract}

brassica, expression patterns of cytokinin oxidase gene fragments during flower and seed development were quantified using realtime PCR. Members of the cytokinin oxidase gene family were isolated and their expression levels monitored in flowers, seeds, leaves and roots. Changes in the level of cytokinin oxidase genes reflect a key role of this gene family in regulation of levels of active cytokinins. 